\title{
Editor Column
}

\section{Dual Perspectives}

Discussion of new, sometimes controversial, ideas is an essential and exciting component of scientific progress. Neuroscientists often have strong views on how a given question should be approached, how results should be interpreted, and how important new findings are for re-evaluating existing data. These discussions frequently occur at small meetings or in casual conversations among experts in a particular area of neuroscience and are not always readily apparent to interested scientists outside that field or to junior scientists just entering a field. To remedy this situation and, we hope, enliven these discussions, the Journal of Neuroscience has begun a new feature called Dual Perspectives, which appears for the first time in this issue.

Dual Perspectives are pairs of articles that present opposing or complementary views about an important issue in neuroscience research. The editors invite two sets of authors to write separate articles discussing their point of view, after which each author is invited to write a brief comment on the companion article. Our first pair of articles discusses different perspectives on the role of the extended amygdala in fear and anxiety, based on human and animal studies. Next month will bring a pair debating the role of TMC channels in hair cell mechanotransduction. We plan to publish a new pair every month or so.

These discussions are meant to be provocative, so neither piece is solicited to be fully balanced across the various viewpoints in the field. We hope that by allowing scientists to take a strong point of view, we will make clear where the points of agreement and disagreement are most profound, and perhaps where future research might clarify the issue. We invite readers of JNeurosci to weigh in on the discussion online at the JNeurosci website by clicking the "Submit a Response" link in the sidebar of the articles and adding your comments and ideas about the issues raised in these Dual Perspectives.

With this new feature, we hope not only to keep JNeurosci at the forefront of discussions of new, interesting, multi-level and controversial issues in neuroscience, but also to highlight voices that are not always heard, including those of younger PIs.

Dual Perspectives join TechSights, short reviews about advantages and pitfalls of novel techniques that are broadly useful to neuroscientists, and ViewPoints, more general mini reviews highlighting current topics in neuroscience, as Featured Articles meant to promote discussion of issues in neuroscience today.

If you have ideas about current controversies in neuroscience that you think should be highlighted in a Dual Perspective article, please contact us at JN_EIC@sfn.org or JN_Features@sfn.org.

\footnotetext{
(D) Marina Picciotto, EiC, JNeurosci

(DTeresa Esch, Features Editor JNeurosci

DOI:10.1523/JNEUROSCI.2247-16.2016
} 